\title{
Arithmetic properties of 5-regular bipartitions
}

\author{
M. S. Mahadeva Naika* and B. Hemanthkumar ${ }^{\dagger}$ \\ Department of Mathematics, Bangalore University \\ Central College Campus, Bengaluru \\ Karnataka 560001, India \\ *msmnaika@rediffmail.com \\ †hemanthkumarb.30@gmail.com
}

Received 15 June 2015

Accepted 21 April 2016

Published 26 October 2016

Let $B_{t}(n)$ denote the number of $t$-regular bipartitions of $n$. In this work, we establish several infinite families of congruences modulo powers of 2 and 5 for $B_{5}(n)$. For example, we find that for all nonnegative integers $n, i$ and $j$ and $r \in\{23,47\}$,

$$
B_{5}\left(2^{2 i+4} \cdot 5^{2 j+1} n+\frac{r \cdot 2^{2 i+1} \cdot 5^{2 j}-1}{3}\right) \equiv 0 \quad\left(\bmod 2^{4}\right) .
$$

Keywords: Partition; regular bipartition; congruence.

Mathematics Subject Classification 2010: 11P83, 05A17

\section{Introduction}

A partition of a positive integer $n$ is a nonincreasing sequence of positive integers whose sum is $n$. We denote the number of partitions of $n$ by $p(n)$.

Ramanujan proved that for every nonnegative integer $n$,

$p(5 n+4) \equiv 0 \quad(\bmod 5), \quad p(7 n+5) \equiv 0(\bmod 7) \quad$ and $\quad p(11 n+6) \equiv 0(\bmod 11)$.

Throughout this paper, we will use the notation

$$
(a ; q)_{\infty}:=\prod_{k=0}^{\infty}\left(1-a q^{k}\right) \text { and } f_{k}:=\left(q^{k} ; q^{k}\right)_{\infty} .
$$

Recall that for an integer $t>1$, a $t$-regular partition is a partition none of whose parts is divisible by $t$. We denote the number of $t$-regular partitions of $n$ by $b_{t}(n)$ and assume $b_{t}(0)=1$ by convention. The generating function for $b_{t}(n)$ satisfies

$$
\sum_{n=0}^{\infty} b_{t}(n) q^{n}=\frac{f_{t}}{f_{1}}
$$


Let $f(a, b)$ be Ramanujan's general theta function given by

$$
f(a, b):=\sum_{n=-\infty}^{\infty} a^{\frac{n(n+1)}{2}} b^{\frac{n(n-1)}{2}} .
$$

In this notation, Jacobi's triple product identity takes the form

$$
f(a, b)=(-a ; a b)_{\infty}(-b ; a b)_{\infty}(a b ; a b)_{\infty} .
$$

Thus,

$$
\begin{aligned}
& \psi(q):=f\left(q, q^{3}\right)=\sum_{n=0}^{\infty} q^{\frac{n(n+1)}{2}}=\frac{\left(q^{2} ; q^{2}\right)_{\infty}}{\left(q ; q^{2}\right)_{\infty}} \\
& \varphi(q):=f(q, q)=\sum_{n=-\infty}^{\infty} q^{n^{2}}=\left(-q ; q^{2}\right)_{\infty}^{2}\left(q^{2} ; q^{2}\right)_{\infty}
\end{aligned}
$$

and

$$
f(-q):=f\left(-q,-q^{2}\right)=\sum_{n=-\infty}^{\infty}(-1)^{n} q^{\frac{n(3 n-1)}{2}}=(q ; q)_{\infty}=f_{1} .
$$

Equality (1.3) is a statement of Euler's famous pentagonal number theorem [1, pp. 9-12]. After Ramanujan, we also define

$$
\chi(q):=\left(-q ; q^{2}\right)_{\infty}
$$

Recently, the arithmetic of $t$-regular partition functions has been studied by a number of authors. Calkin et al. [5] examined the 5-regular partition function modulo 2 and the 13-regular partition function modulo 2 and 3 using the theory of modular forms. Hirschhorn and Sellers [7] obtained stronger results for the 5-regular partition function using only Jacobi's triple product identity. For example, for all $m \geq 0$

$$
b_{5}\left(4 p^{2} m+4 u(p r-7)+1\right) \equiv 0 \quad(\bmod 2),
$$

where $p$ is any prime greater than 3 such that -10 is a quadratic nonresidue modulo $p, u$ is the reciprocal of $24 \operatorname{modulo} p^{2}$, and $r \not \equiv 0(\bmod p)$. By studying $p$-dissection of $f(-q)$ and $\psi(q)$, Cui and $\mathrm{Gu}[6]$ have derived several infinite families of congruences modulo 2 for $b_{t}(n)$, where $t \in\{2,4,5,8,13,16\}$. For example, for any prime $p \geq 5$ such that -10 is a quadratic nonresidue modulo $p$,

$$
b_{5}\left(4 p^{2 \alpha+2} n+\frac{(24 i+7 p) p^{2 \alpha+1}-1}{6}\right) \equiv 0 \quad(\bmod 2)
$$

and

$$
b_{5}\left(4 \cdot 5^{2 \alpha+1} n+\frac{r \cdot 5^{2 \alpha}-1}{6}\right) \equiv 0 \quad(\bmod 2)
$$

for all $\alpha, n \geq 0,1 \leq i \leq p-1$ and $r \in\{31,79\}$. 
A bipartition of $n$ is an ordered pair of partitions $\left(\pi_{1}, \pi_{2}\right)$ such that the sum of all of the parts equals $n$, where $\pi_{1}$ and $\pi_{2}$ are allowed to be the empty partition. Let $p_{-2}(n)$ denote the number of bipartitions of $n$. The generating function for $p_{-2}(n)$ satisfies

$$
\sum_{n=0}^{\infty} p_{-2}(n) q^{n}=\frac{1}{f_{1}^{2}}
$$

Several mathematicians have studied the function $p_{-2}(n)$. For example, Ramanathan [12] established the following analogs of Ramanujan's classical congruences for $p(n)$ (see also [2]):

$$
p_{-2}(5 n+2) \equiv p_{-2}(5 n+3) \equiv p_{-2}(5 n+4) \equiv 0 \quad(\bmod 5) .
$$

For $t>1$, a bipartition is said to be $t$-regular if none of its parts is divisible by $t$. Let $B_{t}(n)$ denote the number of $t$-regular bipartitions of $n$. Then the generating function of $B_{t}(n)$ satisfies

$$
\sum_{n=0}^{\infty} B_{t}(n) q^{n}=\frac{f_{t}^{2}}{f_{1}^{2}}
$$

Lin [8] proved that

$$
B_{4}\left(3^{2 \alpha+2} n+\frac{11 \cdot 3^{2 \alpha+1}-1}{4}\right) \equiv 0 \quad(\bmod 3),
$$

for all $\alpha, n \geq 0$, and using Ramanujan's modular equations of degree seven [9] showed that

$$
B_{7}\left(3^{\alpha+2} n+\frac{5 \cdot 3^{\alpha+1}-1}{2}\right) \equiv 0 \quad(\bmod 3) .
$$

In $[10]$ the same author proved that

$$
B_{13}(9 n+5) \equiv 0 \quad(\bmod 3)
$$

and

$$
B_{13}(3 n+2) \equiv B_{13}(9 n+8) \quad(\bmod 3) .
$$

The aim of this paper is to study the arithmetic properties of 5-regular bipartitions in the spirit of Ramanujan's congruences for the partition function $p(n)$.

The main results of this paper can be stated as follows.

Theorem 1.1. Let $r \in\{29,53,77,101\}$. Then, for all nonnegative integers $\alpha$ and $n$, we have

$$
B_{5}\left(2^{2 \alpha+1} n+\frac{2^{2 \alpha+2}-1}{3}\right) \equiv B_{5}(2 n+1) \quad\left(\bmod 2^{2}\right),
$$


4 M. S. Mahadeva Naika \& B. Hemanthkumar

$$
\begin{aligned}
B_{5}\left(2^{2 \alpha+3} n+\frac{11 \cdot 2^{2 \alpha+1}-1}{3}\right) & \equiv 0 \quad\left(\bmod 2^{2}\right), \\
B_{5}\left(2^{2 \alpha+4} n+\frac{17 \cdot 2^{2 \alpha+1}-1}{3}\right) & \equiv 0 \quad\left(\bmod 2^{2}\right), \\
B_{5}\left(5 \cdot 2^{2 \alpha+4} n+\frac{r \cdot 2^{2 \alpha+1}-1}{3}\right) & \equiv 0 \quad\left(\bmod 2^{2}\right), \\
B_{5}\left(2^{2 \alpha+4} n+\frac{7 \cdot 2^{2 \alpha+2}-1}{3}\right) & \equiv 0 \quad\left(\bmod 2^{2}\right)
\end{aligned}
$$

and

$$
B_{5}\left(2^{2 \alpha+5} n+\frac{13 \cdot 2^{2 \alpha+2}-1}{3}\right) \equiv 0 \quad\left(\bmod 2^{2}\right)
$$

Moreover,

$$
\begin{aligned}
B_{5}( & \left.5 \cdot 2^{2 \alpha+4} n+\frac{5 \cdot 2^{2 \alpha+1}-1}{3}\right) \\
& \equiv B_{5}\left(2^{2 \alpha+5} n+\frac{2^{2 \alpha+2}-1}{3}\right) \\
& \equiv\left\{\begin{array}{lll}
2 & \left(\bmod 2^{2}\right) & \text { if } n=k(3 k+1) / 2 \text { for some } k \in \mathbb{Z} \\
0 & \left(\bmod 2^{2}\right) & \text { otherwise. }
\end{array}\right.
\end{aligned}
$$

Theorem 1.2. For all nonnegative integers $\alpha$ and $n$, we have

$$
\begin{aligned}
B_{5}\left(2^{2 \alpha+2} n+\frac{2^{2 \alpha+2}-1}{3}\right) & \equiv B_{5}(4 n+1) \quad\left(\bmod 2^{3}\right), \\
B_{5}(16 n+15) & \equiv 0 \quad\left(\bmod 2^{3}\right), \\
B_{5}\left(2^{2 \alpha+5} n+\frac{19 \cdot 2^{2 \alpha+2}-1}{3}\right) & \equiv 0 \quad\left(\bmod 2^{3}\right)
\end{aligned}
$$

and

$$
B_{5}\left(2^{2 \alpha+6} n+\frac{23 \cdot 2^{2 \alpha+3}-1}{3}\right) \equiv 0 \quad\left(\bmod 2^{3}\right) .
$$

Theorem 1.3. Let $r \in\{83,107\}$ and $s \in\{31,79\}$. Then, for all nonnegative integers $\alpha, j$ and $n$, we have

$$
\begin{gathered}
\sum_{n=0}^{\infty} B_{5}\left(2^{2 \alpha+4} \cdot 5^{2 j} n+\frac{11 \cdot 2^{2 \alpha+1} \cdot 5^{2 j}-1}{3}\right) q^{n} \equiv 4 f_{1} f_{5}^{2} \quad\left(\bmod 2^{3}\right), \\
B_{5}\left(2^{2 \alpha+4} \cdot 5^{2 j+1} n+\frac{r \cdot 2^{2 \alpha+1} \cdot 5^{2 j}-1}{3}\right) \equiv 0 \quad\left(\bmod 2^{3}\right)
\end{gathered}
$$




$$
\begin{aligned}
B_{5}\left(2^{2 \alpha+4} \cdot 5^{2 j+2} n+\frac{s \cdot 2^{2 \alpha+1} \cdot 5^{2 j+1}-1}{3}\right) & \equiv 0 \quad\left(\bmod 2^{3}\right), \\
\sum_{n=0}^{\infty} B_{5}\left(2^{2 \alpha+5} \cdot 5^{2 j} n+\frac{7 \cdot 2^{2 \alpha+2} \cdot 5^{2 j}-1}{3}\right) q^{n} & \equiv 4 f_{1}^{2} f_{5} \quad\left(\bmod 2^{3}\right), \\
B_{5}\left(2^{2 \alpha+5} \cdot 5^{2 j+1} n+\frac{s \cdot 2^{2 \alpha+2} \cdot 5^{2 j}-1}{3}\right) & \equiv 0 \quad\left(\bmod 2^{3}\right)
\end{aligned}
$$

and

$$
B_{5}\left(2^{2 \alpha+5} \cdot 5^{2 j+2} n+\frac{r \cdot 2^{2 \alpha+2} \cdot 5^{2 j+1}-1}{3}\right) \equiv 0 \quad\left(\bmod 2^{3}\right) .
$$

Theorem 1.4. For all nonnegative integers $\alpha$ and $n$, we have

$$
B_{5}\left(2^{2 \alpha+4} n+\frac{2^{2 \alpha+4}-1}{3}\right) \equiv B_{5}(16 n+5) \quad\left(\bmod 2^{4}\right) .
$$

Theorem 1.5. Let $r \in\{23,47\}$. Then, for all nonnegative integers $\alpha, j$ and $n$, we have

$$
\sum_{n=0}^{\infty} B_{5}\left(2^{\alpha+4} \cdot 5^{2 j+k} n+\frac{23 \cdot 2^{\alpha+1} \cdot 5^{2 j+k}-1}{3}\right) q^{n} \equiv 8 q^{2} f_{1} f_{5}^{14}\left(\bmod 2^{4}\right)
$$

and

$$
B_{5}\left(2^{\alpha+4} \cdot 5^{2 j+1+k} n+\frac{r \cdot 2^{\alpha+1} \cdot 5^{2 j+k}-1}{3}\right) \equiv 0 \quad\left(\bmod 2^{4}\right)
$$

where

$$
k= \begin{cases}0 & \text { if } \alpha \text { is even } \\ 1 & \text { if } \alpha \text { is odd } .\end{cases}
$$

Theorem 1.6. For all nonnegative integers $\alpha$ and $n$, we have

$$
\begin{gathered}
B_{5}(5 n+2) \equiv B_{5}(5 n+3) \equiv B_{5}(5 n+4) \equiv 0 \quad(\bmod 5), \\
B_{5}\left(2^{2 \alpha} n+\frac{2^{2 \alpha}-1}{3}\right) \equiv 2^{\alpha} B_{5}(n) \quad(\bmod 5)
\end{gathered}
$$

and

$$
B_{5}\left(2^{2 \alpha+2} n+\frac{2^{2 \alpha+1} \cdot 5-1}{3}\right) \equiv 0 \quad(\bmod 5) .
$$

Theorem 1.7. Let $r \in\{11,29\}$ and $s \in\{7,13,25\}$. Then, for all nonnegative integers $\alpha$ and $n$, we have

$$
B_{5}\left(5 \cdot 2^{2 \alpha+2} n+\frac{r \cdot 2^{2 \alpha+1}-1}{3}\right) \equiv 0 \quad\left(\bmod 5^{2}\right)
$$

and

$$
B_{5}\left(5^{2} \cdot 2^{2 \alpha+2} n+\frac{5 s \cdot 2^{2 \alpha+1}-1}{3}\right) \equiv 0 \quad\left(\bmod 5^{2}\right) .
$$


The rest of the paper is organized as follows. In Sec. 2 we establish some preliminary results, and prove our main results in Secs. $3-5$.

\section{Preliminaries}

In this section, we give three lemmas which are helpful in proving our main results.

Lemma 2.1. The following 2-dissections hold:

$$
\frac{f_{5}}{f_{1}}=\frac{f_{8} f_{20}^{2}}{f_{2}^{2} f_{40}}+q \frac{f_{4}^{3} f_{10} f_{40}}{f_{2}^{3} f_{8} f_{20}}
$$

and

$$
\frac{f_{1}}{f_{5}}=\frac{f_{2} f_{8} f_{20}^{3}}{f_{4} f_{10}^{3} f_{40}}-q \frac{f_{4}^{2} f_{40}}{f_{8} f_{10}^{2}} .
$$

Proof. Equation (2.1) was proved by Hirschhorn and Sellers in [7] (see also [3]). Replacing $q$ by $-q$ in (2.1) and using the relation

$$
(-q ;-q)_{\infty}=\frac{f_{2}^{3}}{f_{1} f_{4}}
$$

we obtain (2.2).

Lemma 2.2. The following 2-dissections hold:

$$
\begin{aligned}
& f_{1}^{2}=\frac{f_{2} f_{8}^{5}}{f_{4}^{2} f_{16}^{2}}-2 q \frac{f_{2} f_{16}^{2}}{f_{8}}, \\
& \frac{1}{f_{1}^{2}}=\frac{f_{8}^{5}}{f_{2}^{5} f_{16}^{2}}+2 q \frac{f_{4}^{2} f_{16}^{2}}{f_{2}^{5} f_{8}}, \\
& f_{1}^{4}=\frac{f_{4}^{10}}{f_{2}^{2} f_{8}^{4}}-4 q \frac{f_{2}^{2} f_{8}^{4}}{f_{4}^{2}}
\end{aligned}
$$

and

$$
\frac{1}{f_{1}^{4}}=\frac{f_{4}^{14}}{f_{2}^{14} f_{8}^{4}}+4 q \frac{f_{4}^{2} f_{8}^{4}}{f_{2}^{10}}
$$

Proof. Lemma 2.2 is an immediate consequence of dissection formulas of Ramanujan, collected in Berndt's book [4, Entry 25, p. 40].

Lemma 2.3. We have

$$
f_{1} f_{5}^{3}=2 q^{2} f_{4} f_{20}^{3}+f_{2}^{3} f_{10}-2 q^{3} \frac{f_{4}^{4} f_{40}^{2} f_{10}}{f_{2} f_{8}^{2}}-q \frac{f_{2}^{2} f_{10}^{2} f_{20}}{f_{4}}
$$

and

$$
f_{1}^{3} f_{5}=2 q^{2} \frac{f_{4}^{6} f_{40}^{2} f_{10}}{f_{2} f_{8}^{2} f_{20}^{2}}+\frac{f_{4} f_{10}^{2} f_{2}^{2}}{f_{20}}+2 q f_{4}^{3} f_{20}-5 q f_{2} f_{10}^{3} .
$$


Proof. In [11] the authors and Sumanth Bharadwaj employ theta function identities of Ramanujan to show that

$$
\begin{aligned}
& \left(-q ; q^{2}\right)_{\infty}\left(-q^{5} ; q^{10}\right)_{\infty}^{3}-\left(q ; q^{2}\right)_{\infty}\left(q^{5} ; q^{10}\right)_{\infty}^{3}=4 q^{3} \frac{f_{4}^{4} f_{40}^{2}}{f_{2}^{2} f_{8}^{2} f_{10}^{2}}+2 q \frac{f_{2} f_{20}}{f_{4} f_{10}} \\
& \left(-q ; q^{2}\right)_{\infty}\left(-q^{5} ; q^{10}\right)_{\infty}^{3}+\left(q ; q^{2}\right)_{\infty}\left(q^{5} ; q^{10}\right)_{\infty}^{3}=4 q^{2} \frac{f_{4} f_{20}^{3}}{f_{2} f_{10}^{3}}+2 \frac{f_{2}^{2}}{f_{10}^{2}} \\
& \left(-q ; q^{2}\right)_{\infty}^{3}\left(-q^{5} ; q^{10}\right)_{\infty}-\left(q ; q^{2}\right)_{\infty}^{3}\left(q^{5} ; q^{10}\right)_{\infty}=10 q \frac{f_{10}^{2}}{f_{2}^{2}}-4 q \frac{f_{4}^{3} f_{20}}{f_{2}^{3} f_{10}}
\end{aligned}
$$

and

$$
\left(-q ; q^{2}\right)_{\infty}^{3}\left(-q^{5} ; q^{10}\right)_{\infty}+\left(q ; q^{2}\right)_{\infty}^{3}\left(q^{5} ; q^{10}\right)_{\infty}=4 q^{2} \frac{f_{4}^{6} f_{40}^{2}}{f_{2}^{4} f_{8}^{2} f_{20}^{2}}+2 \frac{f_{4} f_{10}}{f_{2} f_{20}} .
$$

Subtracting (2.9) from (2.10), and then using the relation

$$
\left(q ; q^{2}\right)_{\infty}=\frac{f_{1}}{f_{2}}
$$

we obtain (2.7). Similarly, (2.8) follows from (2.11) and (2.12).

\section{Proofs of Theorems 1.1-1.5}

Setting $t=5$ in (1.5) yields

$$
\sum_{n=0}^{\infty} B_{5}(n) q^{n}=\frac{f_{5}^{2}}{f_{1}^{2}}
$$

Combining (2.1) and (3.1), we see that

$$
\sum_{n=0}^{\infty} B_{5}(2 n+1) q^{n}=2 \frac{f_{2}^{3} f_{10} f_{5}}{f_{1}^{5}}
$$

Substituting (2.1) and (2.6) into (3.2), we have

$$
\begin{aligned}
\sum_{n=0}^{\infty} B_{5}(2 n+1) q^{n} & =2 f_{2}^{3} f_{10} \frac{f_{5}}{f_{1}} \frac{1}{f_{1}^{4}} \\
& =2 f_{2}^{3} f_{10}\left(\frac{f_{8} f_{20}^{2}}{f_{2}^{2} f_{40}}+q \frac{f_{4}^{3} f_{10} f_{40}}{f_{2}^{3} f_{8} f_{20}}\right)\left(\frac{f_{4}^{14}}{f_{2}^{14} f_{8}^{4}}+4 q \frac{f_{4}^{2} f_{8}^{4}}{f_{2}^{10}}\right),
\end{aligned}
$$

which implies that

$$
\sum_{n=0}^{\infty} B_{5}(4 n+1) q^{n}=2 \frac{f_{5} f_{2}^{14} f_{10}^{2}}{f_{1}^{13} f_{20} f_{4}^{3}}+8 q \frac{f_{2}^{5} f_{4}^{3} f_{20} f_{5}^{2}}{f_{1}^{10} f_{10}}
$$

By the binomial theorem, it is easy to see that for any positive integer $k$,

$$
\begin{array}{ll}
f_{k}^{2} \equiv f_{2 k} & (\bmod 2), \\
f_{k}^{4} \equiv f_{2 k}^{2} \quad\left(\bmod 2^{2}\right)
\end{array}
$$


and

$$
f_{k}^{8} \equiv f_{2 k}^{4} \quad\left(\bmod 2^{3}\right) .
$$

From (3.6) and (3.4), it follows that

$$
\frac{f_{5} f_{2}^{14} f_{10}^{2}}{f_{1}^{13} f_{20} f_{4}^{3}} \equiv \frac{f_{2}^{2} f_{10}^{2} f_{4} f_{5}}{f_{20} f_{1}^{5}} \quad\left(\bmod 2^{3}\right)
$$

and

$$
\frac{f_{2}^{5} f_{4}^{3} f_{20} f_{5}^{2}}{f_{1}^{10} f_{10}} \equiv f_{4}^{3} f_{20} \quad(\bmod 2)
$$

In view of (3.7) and (3.8), we can rewrite (3.3) as

$$
\sum_{n=0}^{\infty} B_{5}(4 n+1) q^{n} \equiv 2 \frac{f_{2}^{2} f_{10}^{2} f_{4}}{f_{20}} \frac{f_{5}}{f_{1}} \frac{1}{f_{1}^{4}}+8 q f_{4}^{3} f_{20} \quad\left(\bmod 2^{4}\right) .
$$

By (3.5) and (3.9), we see that

$$
\sum_{n=0}^{\infty} B_{5}(4 n+1) q^{n} \equiv 2 \frac{f_{10}^{2} f_{4} f_{5}}{f_{20} f_{1}} \quad\left(\bmod 2^{3}\right) .
$$

Substituting (2.1) and (2.6) into (3.9), we obtain

$$
\begin{aligned}
\sum_{n=0}^{\infty} B_{5}(4 n+1) q^{n} \equiv & 2 \frac{f_{2}^{2} f_{10}^{2} f_{4}}{f_{20}}\left(\frac{f_{8} f_{20}^{2}}{f_{2}^{2} f_{40}}+q \frac{f_{4}^{3} f_{10} f_{40}}{f_{2}^{3} f_{8} f_{20}}\right)\left(\frac{f_{4}^{14}}{f_{2}^{14} f_{8}^{4}}+4 q \frac{f_{4}^{2} f_{8}^{4}}{f_{2}^{10}}\right) \\
& +8 q f_{4}^{3} f_{20}\left(\bmod 2^{4}\right),
\end{aligned}
$$

which yields

$$
\sum_{n=0}^{\infty} B_{5}(8 n+5) q^{n} \equiv 2 \frac{f_{2}^{18} f_{5}^{3} f_{20}}{f_{1}^{15} f_{4}^{5} f_{10}^{2}}+8 \frac{f_{4}^{5} f_{2}^{3} f_{10} f_{5}^{2}}{f_{1}^{10} f_{20}}+8 f_{2}^{3} f_{10} \quad\left(\bmod 2^{4}\right) .
$$

It follows from (3.6) and (3.4) that

$$
\frac{f_{2}^{18} f_{5}^{3} f_{20}}{f_{1}^{15} f_{4}^{5} f_{10}^{2}} \equiv \frac{f_{2}^{2} f_{20} f_{10}^{2} f_{1}}{f_{4} f_{5}^{5}} \quad\left(\bmod 2^{3}\right)
$$

and

$$
\frac{f_{4}^{5} f_{2}^{3} f_{10} f_{5}^{2}}{f_{1}^{10} f_{20}} \equiv f_{4}^{4} \quad(\bmod 2) .
$$

In view of (3.12) and (3.13), we can rewrite (3.11) as

$$
\sum_{n=0}^{\infty} B_{5}(8 n+5) q^{n} \equiv 2 \frac{f_{2}^{2} f_{20} f_{10}^{2}}{f_{4}} \frac{f_{1}}{f_{5}} \frac{1}{f_{5}^{4}}+8 f_{4}^{4}+8 f_{2}^{3} f_{10} \quad\left(\bmod 2^{4}\right),
$$

and by (3.4) we also have

$$
\frac{f_{2}^{2} f_{20} f_{10}^{2} f_{1}}{f_{4} f_{5}^{5}} \equiv \frac{f_{2}^{3} f_{10} f_{5}}{f_{1}^{5}} \quad(\bmod 2)
$$


From the above two identities, we arrive at

$$
\sum_{n=0}^{\infty} B_{5}(8 n+5) q^{n} \equiv 2 \frac{f_{2}^{3} f_{10} f_{5}}{f_{1}^{5}} \quad\left(\bmod 2^{2}\right) .
$$

Using (3.2) and (3.15), we find that for $n \geq 0$,

$$
B_{5}(8 n+5) \equiv B_{5}(2 n+1) \quad\left(\bmod 2^{2}\right) .
$$

By (3.16) and mathematical induction, we find that (1.6) is true.

Using (3.6), we can rewrite (3.2) as

$$
\sum_{n=0}^{\infty} B_{5}(2 n+1) q^{n} \equiv 2 \frac{f_{10}}{f_{2}} f_{1}^{3} f_{5} \quad\left(\bmod 2^{4}\right) .
$$

Employing (2.8) in (3.17) and then extracting the terms involving $q^{2 n+1}$ from both sides of the resulting identity, we arrive at

$$
\sum_{n=0}^{\infty} B_{5}(4 n+3) q^{n} \equiv 4 f_{2}^{3} f_{10} \frac{f_{5}}{f_{1}}-10 f_{5}^{4} \quad\left(\bmod 2^{4}\right),
$$

which implies that

$$
\sum_{n=0}^{\infty} B_{5}(4 n+3) q^{n} \equiv 2 f_{20} \quad\left(\bmod 2^{2}\right) .
$$

From (3.19), it is easy to see that for all $n \geq 0$ and $1 \leq i \leq 4$,

$$
\begin{aligned}
B_{5}(8 n+7) & \equiv 0 \quad\left(\bmod 2^{2}\right), \\
B_{5}(16 n+11) & \equiv 0 \quad\left(\bmod 2^{2}\right), \\
\sum_{n=0}^{\infty} B_{5}(16 n+3) q^{n} & \equiv 2 f_{5} \quad\left(\bmod 2^{2}\right)
\end{aligned}
$$

and

$$
B_{5}(80 n+16 i+3) \equiv 0 \quad\left(\bmod 2^{2}\right) .
$$

Congruences (1.7)-(1.9) follow from (3.20), (3.21), (3.23) and (1.6).

We can also rewrite $(3.9)$ as

$$
\sum_{n=0}^{\infty} B_{5}(4 n+1) q^{n} \equiv 2 \frac{f_{10}^{2} f_{4}}{f_{20} f_{2}^{2}} f_{1}^{3} f_{5}+8 q f_{4}^{3} f_{20} \quad\left(\bmod 2^{4}\right) .
$$

Applying (2.8) in (3.24), then extracting the terms involving $q^{2 n}$ from both sides, we find that

$$
\sum_{n=0}^{\infty} B_{5}(8 n+1) q^{n} \equiv 4 q \frac{f_{20}^{2} f_{2}^{7} f_{5}^{3}}{f_{10}^{3} f_{4}^{2} f_{1}^{3}}+2 \frac{f_{2}^{2} f_{5}^{4}}{f_{10}^{2}} \quad\left(\bmod 2^{4}\right)
$$


and by (3.4) we also have

$$
\frac{f_{2}^{2} f_{5}^{4}}{f_{10}^{2}} \equiv f_{4} \quad(\bmod 2)
$$

In view of (3.25) and (3.26), we have

$$
\sum_{n=0}^{\infty} B_{5}(8 n+1) q^{n} \equiv 2 f_{4} \quad\left(\bmod 2^{2}\right) .
$$

From (3.27) we deduce that

$$
B_{5}(16 n+9) \equiv 0 \quad\left(\bmod 2^{2}\right)
$$

and

$$
B_{5}(32 n+17) \equiv 0 \quad\left(\bmod 2^{2}\right)
$$

for all $n \geq 0$ and

$$
\sum_{n=0}^{\infty} B_{5}(32 n+1) q^{n} \equiv 2 f_{1} \quad\left(\bmod 2^{2}\right) .
$$

Congruences (1.10) and (1.11) follow from (3.28), (3.29) and (1.6).

From (3.22) and (3.30), we see that

$$
\sum_{n=0}^{\infty} B_{5}(80 n+3) q^{n} \equiv \sum_{n=0}^{\infty} B_{5}(32 n+1) q^{n} \equiv 2 f_{1} \quad\left(\bmod 2^{2}\right)
$$

and by (1.3) we have

$$
f_{1}=\sum_{k=-\infty}^{\infty}(-1)^{k} q^{k(3 k-1) / 2} .
$$

Combining (3.31) and (3.32), we deduce that

$$
\begin{aligned}
\sum_{n=0}^{\infty} B_{5}(80 n+3) q^{n} & \equiv \sum_{n=0}^{\infty} B_{5}(32 n+1) q^{n} \\
& \equiv 2 \sum_{k=-\infty}^{\infty} q^{k(3 k-1) / 2}\left(\bmod 2^{2}\right)
\end{aligned}
$$

By (3.33) and (1.6), we find that (1.12) is true.

Invoking (2.2) and (2.6) in (3.14), we obtain

$$
\begin{aligned}
\sum_{n=0}^{\infty} B_{5}(8 n+5) q^{n} \equiv & 2 \frac{f_{2}^{2} f_{20} f_{10}^{2}}{f_{4}}\left(\frac{f_{2} f_{8} f_{20}^{3}}{f_{4} f_{10}^{3} f_{40}}-q \frac{f_{4}^{2} f_{40}}{f_{8} f_{10}^{2}}\right)\left(\frac{f_{20}^{14}}{f_{10}^{14} f_{40}^{4}}+4 q^{5} \frac{f_{20}^{2} f_{40}^{4}}{f_{10}^{10}}\right) \\
& +8 f_{4}^{4}+8 f_{2}^{3} f_{10}\left(\bmod 2^{4}\right),
\end{aligned}
$$


which yields

$$
\sum_{n=0}^{\infty} B_{5}(16 n+5) q^{n} \equiv 2 \frac{f_{1}^{3} f_{10}^{18} f_{4}}{f_{5}^{15} f_{2}^{2} f_{20}^{5}}-8 q^{3} \frac{f_{1}^{2} f_{2} f_{10}^{3} f_{20}^{5}}{f_{4} f_{5}^{10}}+8 f_{2}^{4}+8 f_{1}^{3} f_{5} \quad\left(\bmod 2^{4}\right) .
$$

From (3.6) and (3.4), it follows that

$$
\frac{f_{1}^{3} f_{10}^{18} f_{4}}{f_{5}^{15} f_{2}^{2} f_{20}^{5}} \equiv \frac{f_{2}^{2} f_{4} f_{10}^{2} f_{5}}{f_{20} f_{1}^{5}} \quad\left(\bmod 2^{3}\right)
$$

and

$$
\frac{f_{1}^{2} f_{2} f_{10}^{3} f_{20}^{5}}{f_{4} f_{5}^{10}} \equiv f_{20}^{4} \quad(\bmod 2) .
$$

From (3.35) and (3.36), (3.34) can be rewritten as

$$
\sum_{n=0}^{\infty} B_{5}(16 n+5) q^{n} \equiv 2 \frac{f_{2}^{2} f_{4} f_{10}^{2}}{f_{20}} \frac{f_{5}}{f_{1}} \frac{1}{f_{1}^{4}}-8 q^{3} f_{20}^{4}+8 f_{2}^{4}+8 f_{1}^{3} f_{5} \quad\left(\bmod 2^{4}\right) .
$$

It follows that

$$
\sum_{n=0}^{\infty} B_{5}(16 n+5) q^{n} \equiv 2 \frac{f_{4} f_{10}^{2} f_{5}}{f_{20} f_{1}} \quad\left(\bmod 2^{3}\right) .
$$

In view of (3.10) and (3.38), we deduce that for all $n \geq 0$,

$$
B_{5}(16 n+5) \equiv B_{5}(4 n+1) \quad\left(\bmod 2^{3}\right) .
$$

By (3.39) and mathematical induction, we can deduce (1.13).

Substituting (2.1) and (2.5) in (3.18), we find that

$$
\begin{aligned}
\sum_{n=0}^{\infty} B_{5}(4 n+3) q^{n} \equiv & 4 f_{2}^{3} f_{10}\left(\frac{f_{8} f_{20}^{2}}{f_{2}^{2} f_{40}}+q \frac{f_{4}^{3} f_{10} f_{40}}{f_{2}^{3} f_{8} f_{20}}\right) \\
& -10\left(\frac{f_{20}^{10}}{f_{10}^{2} f_{40}^{4}}-4 q^{5} \frac{f_{10}^{2} f_{40}^{4}}{f_{20}^{2}}\right) \quad\left(\bmod 2^{4}\right),
\end{aligned}
$$

which yields

$$
\sum_{n=0}^{\infty} B_{5}(8 n+7) q^{n} \equiv 4 \frac{f_{2}^{3} f_{20} f_{5}^{2}}{f_{4} f_{10}}+8 q^{2} \frac{f_{20}^{4} f_{5}^{2}}{f_{10}^{2}} \quad\left(\bmod 2^{4}\right) .
$$

Since

$$
\frac{f_{2}^{3} f_{20} f_{5}^{2}}{f_{4} f_{10}} \equiv f_{2} f_{10}^{2} \quad(\bmod 2)
$$

it follows that

$$
\sum_{n=0}^{\infty} B_{5}(8 n+7) q^{n} \equiv 4 f_{2} f_{10}^{2} \quad\left(\bmod 2^{3}\right) .
$$

Congruence (1.14) follows from (3.42). 
Using (3.5), we can rewrite (3.25) as

$$
\sum_{n=0}^{\infty} B_{5}(8 n+1) q^{n} \equiv 4 q \frac{f_{10}^{3} f_{4}^{2}}{f_{2}^{3}} \frac{f_{1}}{f_{5}}+2 \frac{f_{2}^{2}}{f_{10}^{2}} f_{5}^{4} \quad\left(\bmod 2^{4}\right) .
$$

Now, employing (2.2) and (2.5) in (3.43), we see that

$$
\begin{aligned}
\sum_{n=0}^{\infty} B_{5}(8 n+1) q^{n} \equiv & 4 q \frac{f_{10}^{3} f_{4}^{2}}{f_{2}^{3}}\left(\frac{f_{2} f_{8} f_{20}^{3}}{f_{4} f_{10}^{3} f_{40}}-q \frac{f_{4}^{2} f_{40}}{f_{8} f_{10}^{2}}\right) \\
& +2 \frac{f_{2}^{2}}{f_{10}^{2}}\left(\frac{f_{20}^{10}}{f_{10}^{2} f_{40}^{4}}-4 q^{5} \frac{f_{10}^{2} f_{40}^{4}}{f_{20}^{2}}\right) \quad\left(\bmod 2^{4}\right)
\end{aligned}
$$

which implies that

$$
\sum_{n=0}^{\infty} B_{5}(16 n+9) q^{n} \equiv 4 \frac{f_{2} f_{4} f_{10}^{3}}{f_{20}} \frac{1}{f_{1}^{2}}+8 q^{2} f_{2} f_{20}^{3} \quad\left(\bmod 2^{4}\right) .
$$

By (3.44) and (3.4), we find that

$$
\sum_{n=0}^{\infty} B_{5}(16 n+9) q^{n} \equiv 4 f_{2}^{2} f_{10} \quad\left(\bmod 2^{3}\right)
$$

From (3.45) we deduce that

$$
B_{5}(32 n+25) \equiv 0 \quad\left(\bmod 2^{3}\right)
$$

for all $n \geq 0$ and

$$
\sum_{n=0}^{\infty} B_{5}(32 n+9) q^{n} \equiv 4 f_{1}^{2} f_{5} \quad\left(\bmod 2^{3}\right) .
$$

Congruence (1.15) follows from (3.46) and (1.13).

Using (3.6), we can rewrite (3.14) as

$$
\sum_{n=0}^{\infty} B_{5}(8 n+5) q^{n} \equiv 2 \frac{f_{2}^{2} f_{20}}{f_{10}^{2} f_{4}} f_{1} f_{5}^{3}+8 f_{4}^{4}+8 f_{2}^{3} f_{10} \quad\left(\bmod 2^{4}\right) .
$$

Invoking (2.7) in (3.48) and then extracting the terms involving $q^{2 n+1}$ from both sides of the resulting identity, we deduce that

$$
\sum_{n=0}^{\infty} B_{5}(16 n+13) q^{n} \equiv-4 q \frac{f_{2}^{3} f_{10} f_{20}^{2}}{f_{4}^{2}} \frac{f_{1}}{f_{5}}-2 \frac{f_{10}^{2}}{f_{2}^{2}} f_{1}^{4} \quad\left(\bmod 2^{4}\right) .
$$

Substituting (2.2) and (2.5) in (3.49), then extracting the terms involving $q^{2 n+1}$ from both sides, we obtain

$$
\sum_{n=0}^{\infty} B_{5}(32 n+29) q^{n} \equiv-4 \frac{f_{10}^{5} f_{4}}{f_{2} f_{20}} \frac{1}{f_{5}^{2}}+8 \frac{f_{4}^{4}}{f_{2}^{2}} f_{5}^{2} \quad\left(\bmod 2^{4}\right) .
$$


It follows from (3.50) and (3.4) that

$$
\sum_{n=0}^{\infty} B_{5}(32 n+29) q^{n} \equiv 4 f_{2} f_{10}^{2} \quad\left(\bmod 2^{3}\right) .
$$

From (3.51) we see that

$$
B_{5}(64 n+61) \equiv 0 \quad\left(\bmod 2^{3}\right)
$$

for all $n \geq 0$ and

$$
\sum_{n=0}^{\infty} B_{5}(64 n+29) q^{n} \equiv 4 f_{1} f_{5}^{2} \quad\left(\bmod 2^{3}\right)
$$

Congruence (1.16) follows from (3.52) and (1.13).

Identity (3.42) implies that

$$
\sum_{n=0}^{\infty} B_{5}(16 n+7) q^{n} \equiv 4 f_{1} f_{5}^{2} \quad\left(\bmod 2^{3}\right) .
$$

From (1.13) and (3.53), we can easily see that for all integers $\alpha \geq 0$,

$$
\sum_{n=0}^{\infty} B_{5}\left(2^{2 \alpha+6} n+\frac{11 \cdot 2^{2 \alpha+3}-1}{3}\right) q^{n} \equiv 4 f_{1} f_{5}^{2} \quad\left(\bmod 2^{3}\right) .
$$

In view of (3.54) and (3.55), we see that congruence (1.17) is true for $j=0$.

Now suppose that (1.17) holds for some $j \geq 0$, and recall Ramanujan's 5-dissection [13, p. 212]

$$
f_{1}=f_{25}\left(a(q)-q-q^{2} a^{-1}(q)\right)
$$

where $a(q):=\frac{f\left(-q^{15},-q^{10}\right)}{f\left(-q^{20},-q^{5}\right)}$.

Utilizing (3.56) in (1.17) and then extracting the terms involving $q^{5 n+1}$ from both sides of the resulting congruence, we find that

$$
\begin{aligned}
\sum_{n=0}^{\infty} & B_{5}\left(2^{2 \alpha+4} \cdot 5^{2 j}(5 n+1)+\frac{11 \cdot 2^{2 \alpha+1} \cdot 5^{2 j}-1}{3}\right) q^{n} \\
& =\sum_{n=0}^{\infty} B_{5}\left(2^{2 \alpha+4} \cdot 5^{2 j+1} n+\frac{7 \cdot 2^{2 \alpha+1} \cdot 5^{2 j+1}-1}{3}\right) q^{n} \\
& \equiv 4 f_{5} f_{1}^{2} \\
& \equiv 4 f_{5} f_{25}^{2}\left(a^{2}(q)+q^{2}+q^{4} a^{-2}(q)\right)\left(\bmod 2^{3}\right) .
\end{aligned}
$$

Therefore,

$$
\begin{aligned}
\sum_{n=0}^{\infty} & B_{5}\left(2^{2 \alpha+4} \cdot 5^{2 j+1}(5 n+2)+\frac{7 \cdot 2^{2 \alpha+1} \cdot 5^{2 j+1}-1}{3}\right) q^{n} \\
& =\sum_{n=0}^{\infty} B_{5}\left(2^{2 \alpha+4} \cdot 5^{2(j+1)} n+\frac{11 \cdot 2^{2 \alpha+1} \cdot 5^{2(j+1)}-1}{3}\right) q^{n} \\
& \equiv 4 f_{1} f_{5}^{2} \quad\left(\bmod 2^{3}\right) .
\end{aligned}
$$


Thus, (1.17) is true for $j+1$. Hence, by mathematical induction congruence (1.17) holds for all $j \geq 0$, and thanks to (3.56), congruences (1.18) and (1.19) follow easily from (1.17) and (3.57), respectively.

It follows from (3.47) and (1.13) that for all integers $\alpha \geq 0$,

$$
\sum_{n=0}^{\infty} B_{5}\left(2^{2 \alpha+5} n+\frac{7 \cdot 2^{2 \alpha+2}-1}{3}\right) q^{n} \equiv 4 f_{1}^{2} f_{5} \quad\left(\bmod 2^{3}\right),
$$

which is the $j=0$ case of (1.20). The rest of the proof by mathematical induction is similar to that of (1.17), so we omit the details. Congruences (1.21) and (1.22) follow immediately from the proof of (1.20).

Substituting (2.1), (2.6) and (2.8) in (3.37) and then extracting the terms involving $q^{2 n+1}$ from both sides of the resulting identity, we deduce that

$$
\begin{aligned}
\sum_{n=0}^{\infty} B_{5}(32 n+21) q^{n} & \equiv 2 \frac{f_{2}^{18} f_{5}^{3} f_{20}}{f_{1}^{15} f_{4}^{5} f_{10}^{2}}+8 \frac{f_{4}^{5} f_{2}^{3} f_{10} f_{5}^{2}}{f_{1}^{10} f_{20}}-8 q f_{10}^{4}+8 f_{1} f_{5}^{3} \\
& \equiv 2 \frac{f_{2}^{2} f_{20} f_{10}^{2}}{f_{4}} \frac{f_{1}}{f_{5}} \frac{1}{f_{5}^{4}}+8 f_{4}^{4}-8 q f_{10}^{4}+8 f_{1} f_{5}^{3} \quad\left(\bmod 2^{4}\right) .
\end{aligned}
$$

Now, employing (2.2), (2.6) and (2.7) in (3.58) and then extracting the terms which involve $q^{2 n}$, we arrive at

$$
\sum_{n=0}^{\infty} B_{5}(64 n+21) q^{n} \equiv 2 \frac{f_{2}^{2} f_{4} f_{10}^{2} f_{5}}{f_{20} f_{1}^{5}}-8 q^{3} f_{20}^{4}+8 f_{2}^{4}+8 f_{1}^{3} f_{5} \quad\left(\bmod 2^{4}\right) .
$$

By (3.37) and (3.59), we deduce that for all $n \geq 0$,

$$
B_{5}(64 n+21) \equiv B_{5}(16 n+5) \quad\left(\bmod 2^{4}\right) .
$$

Congruence (1.23) follows from (3.60) and mathematical induction.

Invoking (2.3) in (3.40), we see that

$$
\sum_{n=0}^{\infty} B_{5}(8 n+7) q^{n} \equiv 4 \frac{f_{20} f_{2}^{3}}{f_{10} f_{4}}\left(\frac{f_{10} f_{40}^{5}}{f_{20}^{2} f_{80}^{2}}-2 q^{5} \frac{f_{10} f_{80}^{2}}{f_{40}}\right)+8 q^{2} \frac{f_{20}^{4}}{f_{10}} \quad\left(\bmod 2^{4}\right),
$$

which implies that

$$
\sum_{n=0}^{\infty} B_{5}(16 n+15) q^{n} \equiv-8 q^{2} \frac{f_{10} f_{1}^{3} f_{40}^{2}}{f_{2} f_{20}} \equiv 8 q^{2} f_{1} f_{5}^{14} \quad\left(\bmod 2^{4}\right) .
$$

Employing (2.4) in (3.44), then extracting terms of the form $q^{2 n+1}$ and using (3.56), we obtain

$$
\begin{aligned}
\sum_{n=0}^{\infty} B_{5}(32 n+25) q^{n} & \equiv 8 \frac{f_{2}^{3} f_{8}^{2} f_{5}^{3}}{f_{10} f_{4} f_{1}^{4}} \\
& \equiv 8 f_{1}^{14} f_{5} \\
& \equiv 8 f_{5} f_{25}^{14}\left(a(q)-q-q^{2} a^{-1}(q)\right)^{14}\left(\bmod 2^{4}\right)
\end{aligned}
$$


Expanding the right-hand side of (3.62) and then extracting the terms involving $q^{5 n+4}$, we see that

$$
\begin{aligned}
\sum_{n=0}^{\infty} B_{5}(160 n+153) q^{n} & =\sum_{n=0}^{\infty} B_{5}\left(2^{5} \cdot 5 n+\frac{23 \cdot 2^{2} \cdot 5-1}{3}\right) q^{n} \\
& \equiv 8 q^{2} f_{1} f_{5}^{14}\left(\bmod 2^{4}\right) .
\end{aligned}
$$

Employing (2.4) in (3.50), we deduce that

$$
\begin{aligned}
\sum_{n=0}^{\infty} B_{5}(64 n+61) q^{n} & =\sum_{n=0}^{\infty} B_{5}\left(2^{6} n+\frac{23 \cdot 2^{3}-1}{3}\right) q^{n} \\
& \equiv 8 q^{2} \frac{f_{10}^{3} f_{40}^{2} f_{1}}{f_{5}^{4} f_{20}} \equiv 8 q^{2} f_{1} f_{5}^{14}\left(\bmod 2^{4}\right)
\end{aligned}
$$

Utilizing (3.6), we can rewrite (3.37) as

$$
\sum_{n=0}^{\infty} B_{5}(16 n+5) q^{n} \equiv 2 \frac{f_{4} f_{10}^{2}}{f_{2}^{2} f_{20}} f_{1}^{3} f_{5}-8 q^{3} f_{20}^{4}+8 f_{2}^{4}+8 f_{1}^{3} f_{5} \quad\left(\bmod 2^{4}\right) .
$$

Substituting (2.8) in (3.65), we obtain

$$
\sum_{n=0}^{\infty} B_{5}(32 n+5) q^{n} \equiv 4 q \frac{f_{2}^{7} f_{20}^{2} f_{5}^{3}}{f_{4}^{2} f_{10}^{3} f_{1}^{3}}+2 \frac{f_{2}^{2} f_{5}^{4}}{f_{10}^{2}} \quad\left(\bmod 2^{4}\right) .
$$

By (3.25) and (3.66), we see that for $n \geq 0$,

$$
B_{5}(32 n+5) \equiv B_{5}(8 n+1) \quad\left(\bmod 2^{4}\right) .
$$

In view of (1.23), congruence (3.67) implies that for all $\alpha \geq 0$,

$$
B_{5}\left(2^{2 \alpha+5}(20 n+19)+\frac{2^{2 \alpha+4}-1}{3}\right) \equiv B_{5}(8(20 n+19)+1) \quad\left(\bmod 2^{4}\right) .
$$

By (3.63), we rewrite (3.68) as

$$
\sum_{n=0}^{\infty} B_{5}\left(2^{2 \alpha+7} \cdot 5 n+\frac{23 \cdot 2^{2 \alpha+4} \cdot 5-1}{3}\right) q^{n} \equiv 8 q^{2} f_{1} f_{5}^{14} \quad\left(\bmod 2^{4}\right) .
$$

Using (3.6), we can rewrite (3.58) as

$$
\sum_{n=0}^{\infty} B_{5}(32 n+21) q^{n} \equiv 2 \frac{f_{2}^{2} f_{20}}{f_{4} f_{10}^{2}} f_{1} f_{5}^{3}+8 f_{4}^{4}-8 q f_{10}^{4}+8 f_{1} f_{5}^{3} \quad\left(\bmod 2^{4}\right) .
$$

Now, employing (2.7) in (3.70), we deduce that

$$
\sum_{n=0}^{\infty} B_{5}(64 n+53) q^{n} \equiv-4 q \frac{f_{1} f_{2}^{3} f_{10} f_{20}^{2}}{f_{4}^{2} f_{5}}-2 \frac{f_{1}^{4} f_{10}^{2}}{f_{2}^{2}} \quad\left(\bmod 2^{4}\right) .
$$

It follows from (3.49) and (3.71) that for $n \geq 0$,

$$
B_{5}(64 n+53) \equiv B_{5}(16 n+13) \quad\left(\bmod 2^{4}\right) .
$$


Therefore,

$$
B_{5}(256 n+245) \equiv B_{5}(64 n+61) \quad\left(\bmod 2^{4}\right) .
$$

In view of $(1.23),(3.64)$ and (3.72), we find that

$$
\sum_{n=0}^{\infty} B_{5}\left(2^{2 \alpha+8} n+\frac{23 \cdot 2^{2 \alpha+5}-1}{3}\right) q^{n} \equiv 8 q^{2} f_{1} f_{5}^{14} \quad\left(\bmod 2^{4}\right) .
$$

Combining (3.61), (3.63), (3.64), (3.69) and (3.73), we obtain

$$
\sum_{n=0}^{\infty} B_{5}\left(2^{\alpha+4} \cdot 5^{k} n+\frac{23 \cdot 2^{\alpha+1} \cdot 5^{k}-1}{3}\right) q^{n} \equiv 8 q^{2} f_{1} f_{5}^{14} \quad\left(\bmod 2^{4}\right),
$$

which is the $j=0$ case of (1.24).

Now suppose that (1.24) holds for some $j \geq 0$. Using (3.56), we have

$$
\begin{aligned}
\sum_{n=0}^{\infty} & B_{5}\left(2^{\alpha+4} \cdot 5^{2 j+k} n+\frac{23 \cdot 2^{\alpha+1} \cdot 5^{2 j+k}-1}{3}\right) q^{n} \\
& \equiv 8 q^{2} f_{1} f_{5}^{14} \\
& \equiv 8 q^{2} f_{5}^{14} f_{25}\left(a(q)-q-q^{2} a^{-1}(q)\right)\left(\bmod 2^{4}\right) .
\end{aligned}
$$

Then

$$
\begin{aligned}
\sum_{n=0}^{\infty} & B_{5}\left(2^{\alpha+4} \cdot 5^{2 j+k}(5 n+3)+\frac{23 \cdot 2^{\alpha+1} \cdot 5^{2 j+k}-1}{3}\right) q^{n} \\
& =\sum_{n=0}^{\infty} B_{5}\left(2^{\alpha+4} \cdot 5^{2 j+k+1} n+\frac{19 \cdot 2^{\alpha+1} \cdot 5^{2 j+k+1}-1}{3}\right) q^{n} \\
& \equiv 8 f_{5} f_{1}^{14} \\
& \equiv 8 f_{5} f_{25}^{14}\left(a(q)-q-q^{2} a^{-1}(q)\right)^{14}\left(\bmod 2^{4}\right)
\end{aligned}
$$

and therefore

$$
\begin{aligned}
\sum_{n=0}^{\infty} & B_{5}\left(2^{\alpha+4} \cdot 5^{2 j+k+1}(5 n+4)+\frac{19 \cdot 2^{\alpha+1} \cdot 5^{2 j+k+1}-1}{3}\right) q^{n} \\
& =\sum_{n=0}^{\infty} B_{5}\left(2^{\alpha+4} \cdot 5^{2(j+1)+k} n+\frac{23 \cdot 2^{\alpha+1} \cdot 5^{2(j+1)+k}-1}{3}\right) q^{n} \\
& \equiv 8 q^{2} f_{1} f_{5}^{14}\left(\bmod 2^{4}\right) .
\end{aligned}
$$

So (1.24) holds. Also, from (3.75) we see that

$$
\sum_{n=0}^{\infty} B_{5}\left(2^{\alpha+4} \cdot 5^{2 j+k}(5 n)+\frac{23 \cdot 2^{\alpha+1} \cdot 5^{2 j+k}-1}{3}\right) q^{n} \equiv 0 \quad\left(\bmod 2^{4}\right)
$$


and

$$
\sum_{n=0}^{\infty} B_{5}\left(2^{\alpha+4} \cdot 5^{2 j+k}(5 n+1)+\frac{23 \cdot 2^{\alpha+1} \cdot 5^{2 j+k}-1}{3}\right) q^{n} \equiv 0 \quad\left(\bmod 2^{4}\right) .
$$

Congruence (1.25) follows from (3.76) and (3.77). This completes the proof.

\section{Proof of Theorem 1.6}

Congruence (1.26) follows readily from (1.4) and (3.1).

For any positive integer $k$, it is easy to see that

$$
f_{k}^{5} \equiv f_{5 k} \quad(\bmod 5) .
$$

Utilizing (4.1), one can rewrite (3.2) as

$$
\sum_{n=0}^{\infty} B_{5}(2 n+1) q^{n} \equiv 2 \frac{f_{10}^{2}}{f_{2}^{2}} \quad(\bmod 5)
$$

which yields

$$
B_{5}(4 n+3) \equiv 0 \quad(\bmod 5)
$$

for all $n \geq 0$ and

$$
\sum_{n=0}^{\infty} B_{5}(4 n+1) q^{n} \equiv 2 \frac{f_{5}^{2}}{f_{1}^{2}} \quad(\bmod 5) .
$$

It follows from (3.1) and (4.3) that for $n \geq 0$,

$$
B_{5}(4 n+1) \equiv 2 B_{5}(n) \quad(\bmod 5) .
$$

Congruence (1.27) follows from (4.4) and mathematical induction. Replacing $n$ by $4 n+3$ in (1.27) and employing (4.2), we deduce (1.28).

\section{Proof of Theorem 1.7}

One of the Ramanujan's modular equations of degree five [4, p. 259] can be written in the equivalent form

$$
5 \frac{\phi^{2}\left(-q^{5}\right)}{\phi^{2}(-q)}-1=4 \frac{\chi\left(-q^{5}\right)}{\chi^{5}(-q)} .
$$

By manipulating the $q$-products, one can easily arrive at

$$
\phi(-q)=\frac{f_{1}^{2}}{f_{2}}, \quad \chi(-q)=\frac{f_{1}}{f_{2}} .
$$

In the view of (5.1) and (5.2), we see that

$$
4 \frac{f_{5}}{f_{1}^{5}}=5 \frac{f_{5}^{4}}{f_{1}^{4} f_{10} f_{2}^{3}}-\frac{f_{10}}{f_{2}^{5}} .
$$


Thanks to (5.3) and (4.1), we can rewrite (3.2) as

$$
\begin{aligned}
\sum_{n=0}^{\infty} 2 B_{5}(2 n+1) q^{n} & =5 \frac{f_{5}^{4}}{f_{1}^{4}}-\frac{f_{10}^{2}}{f_{2}^{2}} \\
& \equiv 5 f_{1} f_{5}^{3}-\frac{f_{10}^{2}}{f_{2}^{2}}\left(\bmod 5^{2}\right) .
\end{aligned}
$$

From (3.56), (3.1) and (5.4),

$$
\begin{aligned}
\sum_{n=0}^{\infty} 2 B_{5}(2 n+1) q^{n} \equiv & 5 f_{5}^{3} f_{25}\left(a(q)-q-q^{2} a^{-1}(q)\right) \\
& -\sum_{n=0}^{\infty} B_{5}(n) q^{2 n} \quad\left(\bmod 5^{2}\right)
\end{aligned}
$$

Equating the terms involving $q^{10 n+3}, q^{10 n+9}$ and $q^{5 n+1}$ on both sides of (5.5), we find that

$$
B_{5}(20 n+7) \equiv B_{5}(20 n+19) \equiv 0 \quad\left(\bmod 5^{2}\right)
$$

for all $n \geq 0$ and

$$
\begin{aligned}
\sum_{n=0}^{\infty} 2 B_{5}(10 n+3) q^{n} \equiv & -5 f_{5} f_{1}^{3}-\sum_{n=0}^{\infty} B_{5}(5 n+3) q^{2 n+1} \\
\equiv & -5 f_{5} f_{25}^{3}\left(a(q)-q-q^{2} a^{-1}(q)\right)^{3} \\
& -\sum_{n=0}^{\infty} B_{5}(5 n+3) q^{2 n+1}\left(\bmod 5^{2}\right) .
\end{aligned}
$$

Now, equating coefficients of $q^{10 n+2}, q^{10 n+4}$ and $q^{10 n+8}$ in (5.7), we obtain

$$
B_{5}(100 n+23) \equiv B_{5}(100 n+43) \equiv B_{5}(100 n+83) \equiv 0 \quad\left(\bmod 5^{2}\right) .
$$

Employing (2.7) in (5.4) and then extracting the terms involving even powers of $q$, we arrive at

$$
\sum_{n=0}^{\infty} 2 B_{5}(4 n+1) q^{n} \equiv 5\left(2 q f_{2} f_{10}^{3}+f_{1}^{3} f_{5}\right)-\frac{f_{5}^{2}}{f_{1}^{2}} \quad\left(\bmod 5^{2}\right) .
$$

Using (3.1), (3.56) and (4.1) in the above congruence, we obtain

$$
\begin{aligned}
\sum_{n=0}^{\infty} B_{5}(4 n+1) q^{n} \equiv & 5 q f_{2} f_{10}^{3}+2 \sum_{n=0}^{\infty} B_{5}(n) q^{n} \\
\equiv & 5 q f_{10}^{3} f_{50}\left(a\left(q^{2}\right)-q^{2}-q^{4} a^{-1}\left(q^{2}\right)\right) \\
& +2 \sum_{n=0}^{\infty} B_{5}(n) q^{n} \quad\left(\bmod 5^{2}\right) .
\end{aligned}
$$

Thus,

$$
B_{5}(20 n+9) \equiv 2 B_{5}(5 n+2) \quad\left(\bmod 5^{2}\right)
$$


and

$$
B_{5}(20 n+17) \equiv 2 B_{5}(5 n+4) \quad\left(\bmod 5^{2}\right)
$$

for all $n \geq 0$ and

$$
\begin{aligned}
\sum_{n=0}^{\infty} B_{5}(20 n+13) q^{n} \equiv & -5 f_{2}^{3} f_{10}+2 \sum_{n=0}^{\infty} B_{5}(5 n+3) q^{n} \\
\equiv & -5 f_{10} f_{50}^{3}\left(a\left(q^{2}\right)-q^{2}-q^{4} a^{-1}\left(q^{2}\right)\right)^{3} \\
& +2 \sum_{n=0}^{\infty} B_{5}(5 n+3) q^{n}\left(\bmod 5^{2}\right)
\end{aligned}
$$

From (5.12) it follows that

$$
\begin{aligned}
& B_{5}(100 n+33) \equiv 2 B_{5}(25 n+8) \quad\left(\bmod 5^{2}\right), \\
& B_{5}(100 n+73) \equiv 2 B_{5}(25 n+18) \quad\left(\bmod 5^{2}\right)
\end{aligned}
$$

and

$$
B_{5}(100 n+93) \equiv 2 B_{5}(25 n+23) \quad\left(\bmod 5^{2}\right) .
$$

By (5.10), (5.11) and mathematical induction, we see that for all $n \geq 0$ and $\alpha \geq 0$,

$$
B_{5}\left(5 \cdot 2^{2 \alpha} n+\frac{13 \cdot 2^{2 \alpha}-1}{3}\right) \equiv 2^{\alpha} B_{5}(5 n+4) \quad\left(\bmod 5^{2}\right)
$$

and

$$
B_{5}\left(5 \cdot 2^{2 \alpha} n+\frac{7 \cdot 2^{2 \alpha}-1}{3}\right) \equiv 2^{\alpha} B_{5}(5 n+2) \quad\left(\bmod 5^{2}\right) .
$$

Congruence (1.29) follows from (5.16), (5.17) and (5.6).

Now, by (5.13) and mathematical induction, we deduce that

$$
B_{5}\left(5^{2} \cdot 2^{2 \alpha} n+\frac{25 \cdot 2^{2 \alpha}-1}{3}\right) \equiv 2^{\alpha} B_{5}(25 n+8) \quad\left(\bmod 5^{2}\right) .
$$

Also, by (5.14), (5.15) and mathematical induction, we have

$$
B_{5}\left(5^{2} \cdot 2^{2 \alpha} n+\frac{55 \cdot 2^{2 \alpha}-1}{3}\right) \equiv 2^{\alpha} B_{5}(25 n+18) \quad\left(\bmod 5^{2}\right)
$$

and

$$
B_{5}\left(5^{2} \cdot 2^{2 \alpha} n+\frac{35 \cdot 2^{2 \alpha+1}-1}{3}\right) \equiv 2^{\alpha} B_{5}(25 n+23) \quad\left(\bmod 5^{2}\right) .
$$

Congruence (1.30) readily follows from (5.18), (5.19), (5.20) and (5.8). This completes the proof. 


\section{Acknowledgments}

We would like to thank the anonymous referee for his/her careful reading of our manuscript and many helpful comments and suggestions. First author would like to thank DST for financial support through project no. SR/S4/MS:739/11 and second author would like to thank UGC for financial support through JRF, ref. no F.17-58/2008(SA-I).

\section{References}

[1] G. E. Andrews, The Theory of Partitions (Cambridge University Press, Cambridge, 1998).

[2] A. O. L. Atkin, Ramanujan congruences for $p_{-k}(n)$, Canad. J. Math. 20 (1968) 67-78; Corrigendum, ibid. 21 (1968) 256.

[3] N. D. Baruah and K. K. Ojah, Analogues of Ramanujan's partition identities and congruences arising from his theta function and modular equations, Ramanujan $J$. 28 (2012) 385-407.

[4] B. C. Berndt, Ramanujan's Notebooks, Part III (Springer, New York, 1991).

[5] N. Calkin, N. Drake, K. James, S. Law, P. Lee, D. Penniston and J. Radder, Divisibility properties of the 5-regular and 13-regular partition functions, Integers 8 (2008) \#A60.

[6] S. P. Cui and N. S. S. Gu, Arithmetic properties of $l$-regular partitions, Adv. Appl. Math. 51 (2013) 507-523.

[7] M. D. Hirschhon and J. A. Sellers, Elementary proofs of parity results for 5-regular partitions, Bull. Aust. Math. Soc. 81 (2010) 58-63.

[8] B. L. S. Lin, Arithmetic properties of bipartition with even parts distinct, Ramanujan J. 33 (2014) 269-279.

[9] - Arithmetic of the 7-regular bipartition function modulo 3, Ramanujan J. 37(3) (2015) 469-478.

[10] B. L. S. Lin, An infinite family of congruences modulo 3 for 13-regular bipartitions, Ramanujan J. 39(1) (2016) 169-178.

[11] M. S. Mahadeva Naika, B. Hemanthkumar and H. S. Sumanth Bharadwaj, Color partitions identities arising from Ramanujan's theta-functions, Acta Math. Vietnam. doi:10.1007/s40306-016-0170-3.

[12] K. G. Ramanathan, Identities and congruences of the Ramanujan type, Canad. J. Math. 2 (1950) 168-178.

[13] S. Ramanujan, Collected Papers (Cambridge University Press, Cambridge, 1927; Reprinted by Chelsea, New York, 1962; Reprinted by the American Mathematical Society, Providence, RI, 2000). 